\title{
Structure of Doubly Oriented Nylon 6
}

\author{
Fumio Nagatoshi and Tamio Arakawa \\ Fiber and Textile Research Institute, Teijin Limited, \\ Minohara 1, Ibaraki, Osaka, Japan.
}

(Received July 20, 1970)

\begin{abstract}
Changes in the structure of doubly oriented nylon 6 film, which was prepared by cold drawing of a film with low moisture content, was studied by X-ray and birefringence measurements. Theoretical values of birefringence were calculated for $\alpha$ - and $\gamma$-forms of the crystal and were used to analyse the experimental results.

Birefringence data indicated that the transition from paracrystalline to $\alpha$-form crystals with improved orientation of the planes of $\mathrm{CH}_{2}$ zigzag chains should be regarded as important, in addition to the increase in crystallinity by heat treatment. The orientation of hydrogen bonds was not appreciably affected by annealing. X-ray data shc wed that the unstable, pseudo-hexagonal structures in the as drawn film continuously approached the stable $\alpha$-form. It has been concluded that the unstable structure should be related to the $\alpha$-form crystal.
\end{abstract}

KEY WORDS Nylon 6/Double Orientation/X-Ray Diffraction/Birefringence / Theoretical Birefringence / $\alpha$-Structure / $\gamma$-Structure / Heat Treatment / Paracrystalline Structure /

Various modifications of the crystal structure have been reported on nylon 6 in addition to the well-known $\alpha$ - and $\gamma$-structures. ${ }^{1-3}$ These modified structures, which are brought about by quenching the melt, cold drawing etc., are usually not very stable, and can be easily transformed into $\alpha$-structure by heat or steam treatment.

The authors recognized that the structure and properties of these unstable crystalline textures can be effectively studied on doubly oriented films prepared under particular conditions. The present paper deals with the structural changes in these films on heat treatment followed by Xray and birefringence measurements. In order to analyse the birefringence data, theoretical values of birefringence were calculated for both $\alpha$ - and $\gamma$-crystal forms.

\section{EXPERIMENTAL}

\section{Sample Preparation}

Extruded nylon 6 film with a thickness of $500 \mu$ and a density of 1.122 was used. Prior to drawing, sample pieces were kept under constant relative humidities at room temperature over 10 days so that their water contents were controlled at desired levels. The conditioned films were then uniaxially drawn to three times their original lengths using a manually operated drawing machine. The drawn films were then heat set in a thermostated air oven for $30 \mathrm{~min}$ while they were fixed on the drawing frame. For heat treatment above $200^{\circ} \mathrm{C}$, the films were first annealed at $160^{\circ} \mathrm{C}$ for $30 \mathrm{~min}$ on the drawing frame, and were then sealed in glass ampules under vacuum. They were then immersed in a thermostated bath for a prescribed period. Steam treatment was carried out on the drawn sample pieces which were heat-treated at $160^{\circ} \mathrm{C}$ for $30 \mathrm{~min}$. The samples were sealed in ampules with adequate amounts of water, and thereafter the ampules were immersed in a bath controlled at $140^{\circ} \mathrm{C}$ for $1 \mathrm{hr}$. To prepare specimens with $\gamma$-structure, the film pieces were immersed in an $1.25 \mathrm{~N} \mathrm{I}_{2}-\mathrm{KI}$ aqueous solution for five days and then kept in a $20 \% \quad \mathrm{Na}_{2} \mathrm{~S}_{2} \mathrm{O}_{3}$ aqueous solution to remove the absorbed iodine.

\section{X-Ray Measurements}

X-ray diffraction was performed using a Rigaku Denki RU III difractometer. Copper $\mathrm{K}_{\alpha}$ radiation was used with a $\mathrm{Ni}$ filter. Most of the measurements were carried out with the incident beam parallel to the drawing direction (end view), since the orientation characteristics 


\section{F. Nagatoshi and T. Arakawa}

of the doubly oriented films are demonstrated most satisfactorily in the diffraction patterns thus obtained.

\section{Birefringence}

The birefringence measurements ware carried out with a polarized microscope with a Berek compensator attached. Since the films were doubly oriented, each specimen required two measurements, that is, with the incident light perpendicular to the film surfaces $\left(\Delta n_{\text {through }}\right)$, and with the incident light parallel to the film surfaces but perpendicular to the drawing direction $\left(\Delta n_{\text {edge }}\right)$.

\section{Calculation of Birefringences for $\alpha$ - and $\gamma$-Struc-} tures

Theoretical values of birefringence were calculated for $\alpha$ - and $\gamma$ - forms of nylon 6 crystals following the normal procedure. ${ }^{4}$ Structural parameters presented by Bunn and coworkers ${ }^{5}$ were employed for the $\alpha$-form, while the data by Arimoto, et al., ${ }^{6}$ were used for the $\gamma$-form. In both cases, $X$-and $Y$-axes were chosen parallel to the crystalline $a$ - and $b$-axes respectively, while the $Z$-axis was perpendicular to the $X-Y$ plane. Assumptions made for the $\alpha$-structure are as follows:

(1) All $\mathrm{C}-\mathrm{H}$ bonds are perpendicular to $Y$-axis, with the bond angle, $\angle \mathrm{HCH}$, of $109^{\circ} 28^{\prime}$.

(2) The hydrogen atom on an amide linkage exists on the linear line connecting $\mathrm{N}$ and $\mathrm{O}$ atoms in the hydrogen bond formation.

In the case of the $\gamma$-form, the coordinates of the hydrogen atoms on carbon atoms were determined assuming the tetrahedral bond angles for carbon atoms. The location of the amide hydrogen atoms was assumed to be on the connecting line between $\mathrm{N}$ and $\mathrm{O}$ atoms linked by the hydrogen bond. The values of the bond polarizabilities used are shown in Table $I$ together with their origins. The resulting chain

Table I. Bond polarizabilities

\begin{tabular}{lccc}
\hline Bond & $\boldsymbol{P}_{L}, \times 10^{25} \mathrm{~cm}^{3}$ & $\boldsymbol{P}_{T}, \times 10^{25} \mathrm{~cm}^{3}$ & References \\
\hline C-C & 9.68 & 2.63 & 13 \\
C-H & 7.9 & 5.8 & 14 \\
N-H & 5.8 & 8.4 & 14 \\
N-C & 13.8 & 2.2 & 4 \\
C $=$ O & 19.9 & 7.5 & 14 \\
\hline
\end{tabular}

Table II. The chain polarizability and refractive index of the $\alpha$-form

\begin{tabular}{ccc}
\hline Direction & $\begin{array}{c}\text { Chain polarizability } \\
\text { per monomer unit, } \\
\times 10^{25} \mathrm{~cm}^{3}\end{array}$ & $\begin{array}{c}\text { Refractive } \\
\text { index }\end{array}$ \\
\hline$X$-axis & 126.16 & 1.6049 \\
$Y$-axis & 131.62 & 1.6378 \\
$Z$-axis & 105.78 & 1.4892 \\
\hline
\end{tabular}

Table III. Chain polarizability and refractive index of the $\gamma$-form

\begin{tabular}{ccc}
\hline Direction & $\begin{array}{c}\text { Chain polarizability } \\
\text { per monomer unit, } \\
\times 10 \mathrm{~cm}\end{array}$ & $\begin{array}{c}\text { Refractive } \\
\text { index }\end{array}$ \\
\hline$X$-axis & 115.49 & 1.5141 \\
$Y$-axis & 128.91 & 1.5870 \\
$Z$-axis & 118.78 & 1.5316 \\
\hline
\end{tabular}

polarizabilities and the refractive indices are given in Tables II and III. Thus the theoretical birefringence values for the $\alpha$-form are $\Delta n_{Y-X}$ $=0.033, \Delta n_{Y-Z}=0.149$ and $\Delta n=0.091$, and those for the $\gamma$-form are $\Delta n_{Y-Z}=0.073, \Delta n_{Y-Z}$ $=0.055$ and $\Delta n=0.064$. Here $\Delta n$ is the birefringence in the usual sense and is equal to $n_{Y}-\left(n_{X}+n_{Z}\right) / 2$. It should be noted that Morgan' has estimated $\Delta n=0.073$ for the ideally oriented nylon 6 fiber containing $\alpha$-crystal by extrapolating the birefringence data obtained by Fujino, et al. ${ }^{8}$

\section{Results and Discussion}

Stretchability of polyamides depends considerably on their moisture content. We studied the relation between the drawing conditions and the structure of the resulting samples using low crystalline, extruded nylon 6 film. At a drawing rate of $100 \%$ per minute at $25^{\circ} \mathrm{C}$, necking was observed when the undrawn films had a water content of less than $2.7 \%$. A typical X-ray diffraction pattern (end view) of the as drawn sample is shown in Figure 1, designated "untreated" (water content at drawing $=0 \%$ ). Although the pattern suggests a seemingly hexagonal packing of the molecules in the drawn film, precise measurement of the lattice spacings for the diffraction spots (arcs) has shown this is not the case. Moreover, as shown in Figure 1, the diffraction pattern of the sample was observed 
Structure of Doubly Oriented Nylon 6

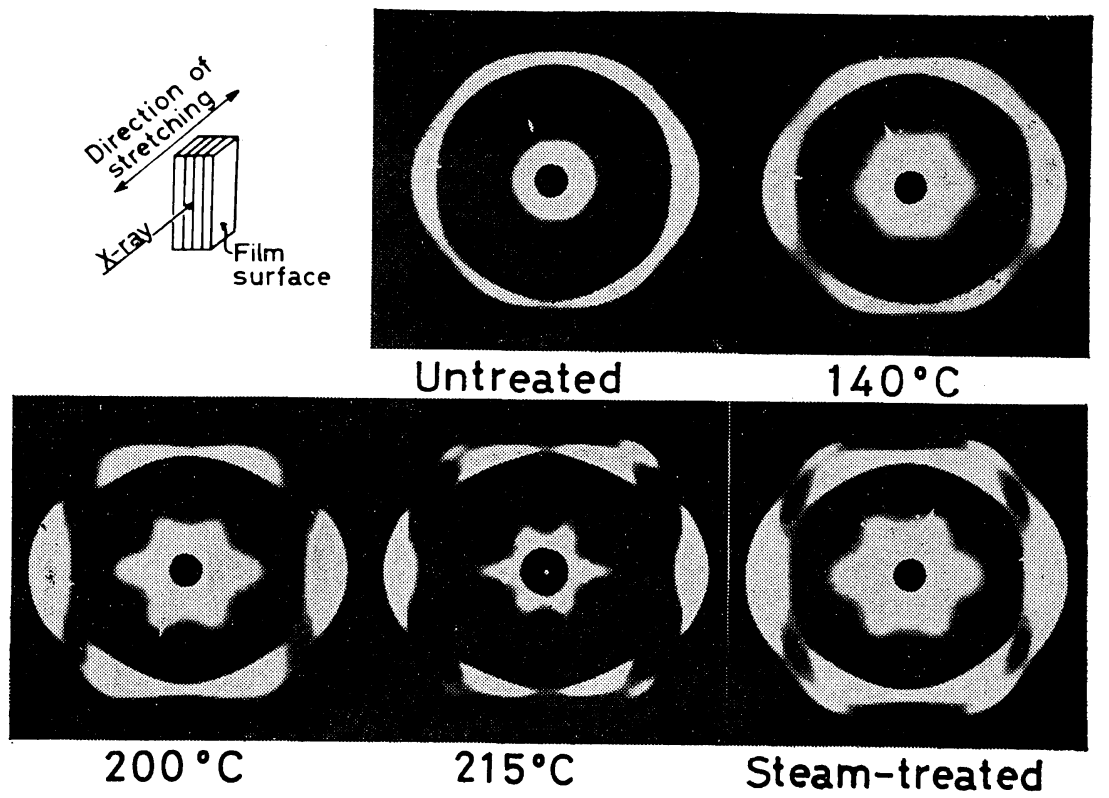

Figure 1. X-ray diffraction patterns (end views) of doubly oriented films treated under various conditions.
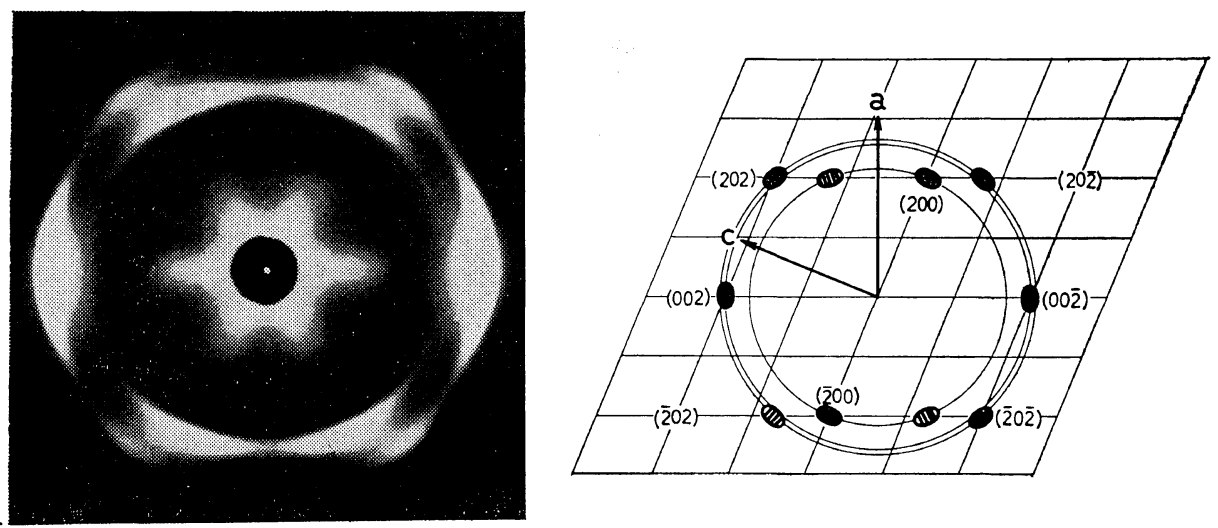

Figure 2. X-ray diffraction pattern (end view) of the doubly oriented film heat-treated at $215^{\circ} \mathrm{C}$, and the corresponding reciprocal lattice. The $b$-axis is perpendicular to the $a c$ plane.

to approach that of the $\alpha$-form crystal with increasingly severe heat or steam treatment (Figure 1). Figure 2 shows the end view diffraction pattern of a doubly oriented film heattreated at $215^{\circ} \mathrm{C}$ for $1 \mathrm{hr}$ and the corresponding reciprocal lattice. The unit cell dimensions are: $a=9.56 \AA, b=17.24 \AA$ (fiber axis), $c=8.01 \AA$, and $\beta=67.5$. The obviously good correspondence between the diffraction pattern and the reciprocal lattice indicates that the $a b$ plane, which corresponds to the plane of the $\mathrm{CH}_{2}$ zigzag chain, is oriented parallel to the film surface. This finding is the same as in the case of the doubly oriented film prepared by roll pressing. ${ }^{9,10}$

What then will be the course of the structural change which takes place in heat treatment of the as drawn film? Depicted in Figure 3 is the change in lattice spacings corresponding to (200) and (002) diffractions in Figure 2 with temper- 


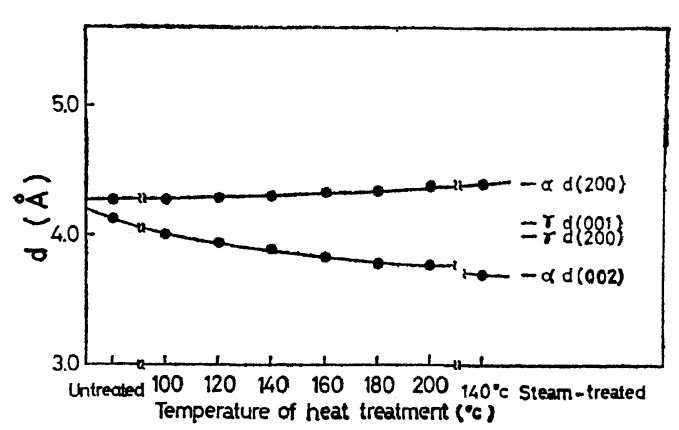

Figure 3. Change of lattice spacings with change in temperature of heat treatment for doubly oriented film.

ature of heat treatment. The four bars in Figure 3 represent the reported values of the lattice spacings for $\alpha$ - and $\gamma$-structures. ${ }^{5,6}$ Results in Figures 1 and 3 suggest that the seemingly hexagonal packing of the molecules in the as drawn film is closely related to the $\alpha$-structure. The gradual change of crystallographic data in Figures 1 and 3 indicates that the structural change by heat treatment is limited to presumably local rearrangement of segments and that the transition from the unstable structure in as drawn film to the $\alpha$-structure in the annealed sample is continuous rather than discrete.

The same conclusion has also been drawn from the birefringence observation. If the crystallographic $a b$ plane is parallel to the film surface, $\Delta n_{\text {through }}$ and $\Delta n_{\text {edge }}$ will correspond to $\Delta n_{Y-Z}$ and $\Delta n_{Y-Z}$ respectively in the case where the doubly oriented specimen is fully crystal-

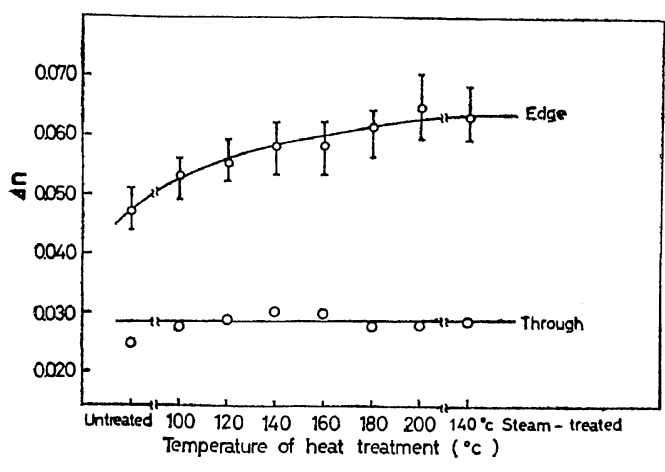

Figure 4. Change of birefringence of double oriented film with change in temperature of heat treatment. lized. Our theoretical calculation has resulted in $\Delta n_{Y-X}=0.033$ and $\Delta n_{Y-Z}=0.149$ for the $\alpha$ structure. The dependence of $\Delta n_{\text {through }}$ and $\Delta n_{\text {edge }}$ on the heat treatment conditions is illustrated in Figure 4 for the doubly oriented film. Although $\Delta n_{\text {through }}$ remains slightly below the theoretical values of $\Delta n_{Y-X}, \Delta n_{\text {edge }}$ increases markedly with increasingly severe heat treatment. At the same time, $\Delta n_{\text {edge }}>\Delta n_{\text {through }}$ is satisfied for all the samples. The increase of $\Delta n_{\text {edge }}$ by heat treatment can be understood in terms of the crystallinity increase. Our previous study $^{11}$ and other studies ${ }^{12}$ on annealing of oriented polymers have shown that the defects in as drawn samples tend to accumulate on annealing to form amorphous regions, while crystalline regions become more crystalline with their crystallite orientation preserved. Apparently both $\Delta n_{\text {edge }}$ and $\Delta n_{\text {through }}$ increase with the increase in crystallinity, and will decrease with increasing amorphous regions. As far as we deal with the two phase model, therefore, it will be difficult to explain the data in Figure 4. Introduction of " $\alpha$-paracrystalline regions", 3 or the regions where molecular chains are in planar conformation parallel to the orientation direction ( $b$-axis) but the planes are in variable equilibrium positions between $a$ - and $c$-axes, can explain our birefringence observations satisfactorily. In these regions $\Delta n_{\text {edge }}<\Delta n_{Y-Z}$ and $\Delta n_{\text {through }}>\Delta n_{Y-X}$ must hold, since we know that $\Delta n_{Y-Z} \gg \Delta n_{Y-X}$.

When the $a b$ planes of the $\alpha$-paracrystals tend to orient parallel to the film surface, the value of $\Delta n_{\text {edge }}$ is expected to increase, since it is to approach $\Delta n_{Y-Z}$. On the other hand, $\Delta n_{\text {through }}$ should decrease under the same conditions, as it is to be related with $\Delta n_{Y-X}$. If the increases in the crystallinity and the orientation of the $a b$ planes take place simultaneously with heat treatment, therefore, the overall effect will be a remarkable increase in $\Delta n_{\text {edge }}$ but a relatively small change in $\Delta n_{\text {through }}$, which is exactly what has been observed in our experiment. In order to investigate the structural change of the as drawn, doubly oriented film on annealing, three specimens with different thermal history were treated with iodine, followed by desorption with sodium thiosulphate solution. They were as drawn (untreated) film, the same drawn film 

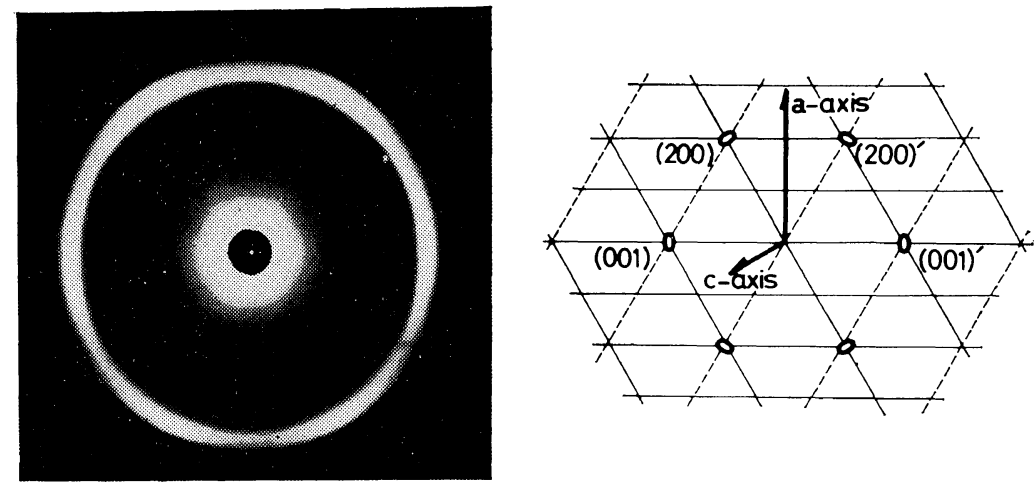

Figure 5. X-ray diffraction pattern (end view) of doubly oriented film with $\gamma$ structure, and the corresponding reciprocal lattice. The $b$-axis is perpendicular to the ac plane.

heat-treated at $160^{\circ} \mathrm{C}$ for $1 \mathrm{hr}$, and the same film steam-treated at $140^{\circ} \mathrm{C}$ for $1 \mathrm{hr}$. The end view X-ray diffraction patterns of these samples turned out identical, as shown in Figure 5. The corresponding reciprocal lattice is also illustrated in the figure. The unit cell dimensions used are: monoclicic, $a=9.33 \AA, b=16.88 \AA$ (fiber axis), $c=4.78 \AA$, and $\beta=121^{\circ}$. In these $\gamma$ structure samples, therefore, the crystallographic $a b$ plane, or the molecular zigzag plane, is alligned parallel to the film surfaces. Since it has been proven by Arimoto ${ }^{15}$ that the allignment of backbone zigzag planes of nylon 6 crystals do not change by the $\alpha \rightarrow \gamma$ transformation, the above result indicates that the planes of the $\mathrm{CH}_{2}$ zigzag chain in the crystals remain unchanged regardless of the heat treatment conditions. The birefringence data for these samples are listed in Table IV. It is clearly seen from the table that $\Delta n_{\text {through }}$ is larger than $\Delta n_{\text {edge }}$ in all cases, which is opposite to the samples with $\alpha$-structure. If the crystallographic $a b$ plane is parallel to the film surface with the $b$-axis oriented in the direction of drawing, $\Delta n_{\text {through }}$ and $\Delta n_{\text {edge }}$ should correspond respectively to $\Delta n_{Y-X}$ and $\Delta n_{Y-Z}$ in the preceding section. The

Table IV. Birefringence of $\gamma$-type samples

\begin{tabular}{lccc}
\hline & $\begin{array}{c}\text { Sample from } \\
\text { untreated film }\end{array}$ & $\begin{array}{c}\text { Sample from Sample from } \\
160^{\circ} \mathrm{C} \text { heat- } \\
\text { treated film }\end{array}$ & $\begin{array}{c}\text { steam } \\
\text { treated film }\end{array}$ \\
\hline$n_{\text {through }}$ & 0.0467 & 0.0500 & 0.0431 \\
$n_{\text {edge }}$ & 0.0368 & 0.0296 & 0.0349 \\
\hline
\end{tabular}

theoretical values of $\Delta n_{Y-X}=0.073$ and $\Delta n_{Y-Z}$ $=0.055$ agree, therefore, rather closely with the above observations. From the above discussion, the following model seems most acceptable for the structural change of the nylon 6 film during drawing and subsequent annealing. By neck drawing, the molecular chains will be oriented in the direction of stretching. At the same time, the plane of $\mathrm{CH}_{2}$ zigzag chains also tends to be oriented parallel to the film surface. The mechanism of the latter structural change was fully discussed in a recent publication. ${ }^{16}$

The orientation of the planes of $\mathrm{CH}_{2}$ zigzag chains is improved by annealing and as a result $\Delta n_{\text {edge }}$ increases considerably. Better alignment of the zigzag chains within hydrogen bonded sheets will reduce the van der Waals repulsion force between the sheets resulting in the decrease in (002) spacing as shown in Figure 3. The parallel increase in (200) spacing is probably due to the increase in $\beta$ of the unit cell from $c a$. $60^{\circ}$ to $67.5^{\circ}$ of the $\alpha$-crysal. It is clear, therefore, that the unstable, pseudo-hexagonal molecular packing in the as drawn film is closely related to the $\alpha$-structure.

\section{REFERENCES}

1. C. Ruscher, V. Gröbe, and H. Versäumer, Faserforsch. u. Textiltech., 12, 214 (1961).

2. M. Tsuruta, H. Arimoto, and M. Ishibashi, Kobunshi Kagaku (Chem. High Polymers), 15, 619 (1958).

3. L. G. Roldan and H. S. Kaufman, J. Polym. 


\section{F. Nagatoshi and T. Arakawa}

Sci., Part B, 1, 603 (1963).

4. C. W. Bunn, "Chemical Crystallography", Clarendon Press, London, 1961.

5. D. R. Holmes, C. W. Bunn, and D. J. Smith, J. Polym. Sci., 17, 159 (1956).

6. H. Arimoto, M. Ishibashi, and M. Hirai, $J$. Polym. Sci., Part A, 3, 317 (1965).

7. H. M. Morgan, Text. Res. J., 32, 886 (1962).

8. K. Fujino, H. Kawai, T. Horino, and K. Miyazawa, Text. Res. J., 26, 852 (1956).

9. T. Aihara, O. Yoshizaki, and O. Nagai, Kobunshi Kagaku (Chem. High Polymers), 20450 (1963).

10. H. Arimoto, Kobunshi Kagaku (Chem. High Polymers), 19, 101 (1962).
11. T. Arakawa, N. Arai, and F. Nagatoshi, $J$. Polym. Sci., Part A-2, 7, 1461 (1969).

12. E. W. Fischer, H. Goddar, and G. F. Schmidt, presented at the Natick Conference on Polymer Structure and Mechanical Properties, Natick, Mass., 1967.

13. C. W. Bunn and R. de P. Daubeny, Trans. Faraday Soc., 50, 1173 (1954).

14. K. G. Denbigh, Trans. Faraday Soc., 36, 936 (1940).

15. H. Arimoto, J. Polym. Sci., Part A, 2, 2283 (1964).

16. T. Okabe, K. Miyasaka, and K. Ishikawa, Kobunshi Kagaku (Chem. High Polymers), 27, 350 (1970). 